\title{
Numerical Study of Plasticity Effects in Uniform Residual Stresses Measurement by Ring-Core Technique
}

\author{
H. Heidary ${ }^{*}$, M. Sadri ${ }^{2}$, N. Zarif Karimi ${ }^{3}$, C. Fragassa ${ }^{3}$ \\ ${ }^{1}$ Department of Mechanical Engineering, Tafresh University, Tafresh, Iran \\ e-mail: heidary@tafreshu.ac.ir; \\ ${ }^{2}$ Department of Mechanical Engineering, Zanjan University, Zanjan, Iran \\ e-mail: mohsen.sadri7@gmail.com \\ ${ }^{3}$ Department of Industrial Engineering, University of Bologna, Bologna, Italy \\ e-mail: navid.zarif@unibo.it \\ e-mail: cristiano.fragassa@unibo.it. \\ *corresponding author
}

\begin{abstract}
The Ring-Core method is a mechanical technique used to calculate the surface residual stresses in a material. In this paper, plasticity effects on the calculated results of the Ring-Core technique were studied by FEM analysis. For this purpose, the coefficients were obtained from an elastic finite element modeling. Then, the execution of the Ring-Core technique using bilinear elasticplastic behavior for the material was simulated and finally the relaxed strains due to ring core milling were obtained. The plastic error due to yielding was calculated by comparing the applied stress and calculated stress from the FE simulation. Also, by using the prepared FE model, the effects of various parameters like state of loading, ring geometry and tangent modulus were investigated. Based on the obtained results, a suitable range for the ring diameter was proposed in order for achieves accurate results.
\end{abstract}

Keywords: Plasticity, residual stress measurement, ring-core, uniform stress

\section{Introduction}

Residual stresses (RS) were developed from different mechanical or thermal operations such as welding, forging, and machining (Withers and Bhadeshia 2001). Residual stresses can cause brittle fracture, buckling, and SCC so determining and measuring have an important role in structure condition assessments. (Rossini 1996, Dassisti et al. 2012).

Hole-Drilling (HD) and Ring-Core (RC) techniques can be used, as a mechanical method, to calculate RS in metallic structures (Rendler and Vigness 1966, Procter and Beaney 1982, Nobre, Kornmeier et al. 2000). These are a semi-destructive experimental method and based on RS relieving due to the removing special form of material from the component surface and monitoring the relaxed strains. In the RC method, an annular groove is milled on the component surface and relived strains are obtained by using strain gauges installed on the center of the ring. The original RS is evaluated based on relieved strain analyses, using equations based on the linear elastic theory (Beaney and Procter 1974). 
In this paper, plasticity error in a uniform RS measurement is investigated by the RC technique using numerical analysis. For this aim, the needed coefficients were obtained from an elastic finite element modeling. Then, the RC technique using bilinear elastic-plastic behavior for the material was simulated and the relaxed strains due to RC milling were obtained. The plastic error due to yielding is evaluated by comparing applied stress and calculated stress from the FE simulation. Also, by using the prepared FE model, the effects of various parameters like state of loading, and tangent modulus have been investigated. Based on the obtained results, a limitation of ring diameter is suggested to obtain a good precision.

\section{Principle and theory of RC strain gauge method}

The RC method is a mechanical technique used to calculate the surface residual stresses in a material. Mechanical techniques, which involve removing material and monitoring strain relaxation, can often provide the only means of determining deep stresses in components. The introduction of a ring into a residually stressed body changes the stresses distribution around the ring. This occurs because shear and normal stresses on new subsisted surface are zero and any changes of stresses field causing the local strains in the bordering region. Mechanical stresses relaxation's principles for RS measurement, first suggested by Mathar, in this manner is used for the development of the RC technique (Mathar 1934).

In the most practical applications of this method, the ring is blind with a depth about equal to its diameter that is small compared to the thickness of the test specimen. Unfortunately, the analysis of blind-ring geometry is adequately complicated, so that no closed-form solution is accessible in this case for direct calculation of the residual stresses from the obtained strains. However, for a simpler form of a through-thickness ring in an infinite plate with a uniform RS distribution, a solution can be obtained based on the theory of elasticity. Thus, the first relation between relived strain and original residual stresses will be derived for the through-ring geometry and consequently will be devoted for application to blind rings.

\subsection{Through-ring stress and strain analysis}

A flat uniform surface area away from edges and other irregularities is chosen by linear elastic behavior of the material as the test specimen of interest.

The initial stress state at any point $\mathrm{P}(\mathrm{R}, \alpha)$ in polar coordinates is:

$$
\begin{aligned}
& \sigma_{r}{ }^{\prime}=\frac{\sigma_{x}}{2}(1+\cos 2 \alpha) \\
& \sigma_{\theta}{ }^{\prime}=\frac{\sigma_{x}}{2}(1-\cos 2 \alpha) \\
& \tau_{r \theta}{ }^{\prime}=\frac{-\sigma_{x}}{2} \sin 2 \alpha
\end{aligned}
$$

Figure 1 shows the same area of the plate after a through ring has been created. 


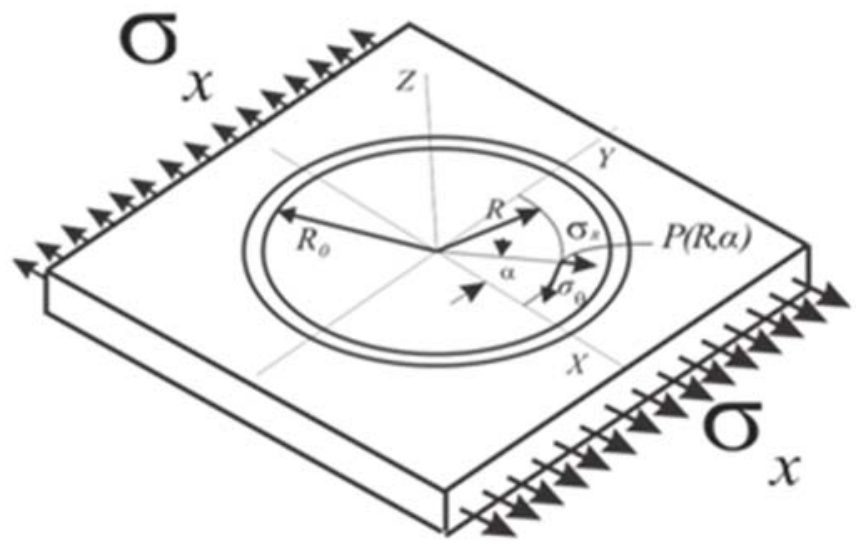

Fig. 1. A through ring on the uni-axial stressed plate

In the created island area, which is completely separated from the surrounding region by the ring, all stress components must be zero everywhere on the ring surface and each point like $\mathrm{P}$.

$$
\sigma_{r}^{\prime \prime}=\sigma_{\theta}^{\prime \prime}=\tau_{r \theta}{ }^{\prime \prime}=0
$$

Subtracting the initial stresses state from the current state gives stress relaxation or stresses varying at point $P(R, \alpha)$ due to ring cutting. That is:

$$
\begin{aligned}
& \Delta \sigma_{r}=\sigma_{r}^{\prime \prime}-\sigma_{r}^{\prime}=\frac{-\sigma_{x}}{2}(1+\cos 2 \alpha) \\
& \Delta \sigma_{\theta}=\sigma_{\theta}^{\prime \prime}-\sigma_{\theta}^{\prime}=\frac{-\sigma_{x}}{2}(1-\cos 2 \alpha)
\end{aligned}
$$

With homogeneous, isotropic and linear-elastic behavior assumption for the plate material and using Hooke's law for biaxial stresses state, the strain in the radial direction of the point $\mathrm{P}$ can be written as bellow:

$$
\begin{aligned}
& \varepsilon_{r}=\left(\frac{1}{E}\right)\left(\Delta \sigma_{r}-v \Delta \sigma_{\theta}\right)=\sigma_{x}(A+B \cos 2 \alpha) \\
& A=\frac{v-1}{2 E} \quad B=\frac{-(v+1)}{2 E}
\end{aligned}
$$

\subsection{Blind-ring stress and strain analysis}

The introduction of a blind ring on a plate with plane stress state generates a very complex local stress distribution and there has been no available exact solution for this until now and no progress has been made so far towards its solution. Fortunately, it has been demonstrated by various numerical studies that stresses distribution on the plate surface is closely similar to the plate with through-ring in nature and condition of stress distribution. Thus, the relieved strains maybe still estimated in the manner described by Eq. (4).

It follows that the equations are also usable to the blind ring implementation when new appropriate coefficients $\bar{A}$ and $\bar{B}$ are used instead of previous confident. Since $\bar{A}$ and $\bar{B}$ could not be derived from the analytical approach directly, they can just be calculated experimentally or numerically. Compared to the through-ring coefficients, blind-ring coefficients involve dimensionless ring depth, $H / D_{I}$ as shown in Fig. 2 and these coefficients can be expressed as: 


$$
\bar{A}=\bar{A}\left(E, v, \frac{H}{D_{I}}\right), \quad \bar{B}=\bar{B}\left(E, v, \frac{H}{D_{I}}\right)
$$

Therefore, Eq. (4) can be rewritten as below and can be used for evaluation of residual stresses by the RC technique:

$$
\varepsilon_{r}=\bar{A}\left(\sigma_{x}+\sigma_{y}\right)+\bar{B}\left(\sigma_{x}-\sigma_{y}\right) \cos 2 \alpha
$$

According to FE investigations, the effect of dimensionless parameter $D_{O} / D_{I}$ is ignorable. Also $\sigma_{x}, \sigma_{y}$ and $\alpha$ are unknown quantities and should be determined as below.

Three independent measurements of $\varepsilon_{r}$ are necessary to solve the equations. The ordinary process for measuring the relieved strains is mounting three resistance strain gauges in rosette form on the center of ring before cutting. Such a rosette and position of mounting are shown schematically in Fig. 2.
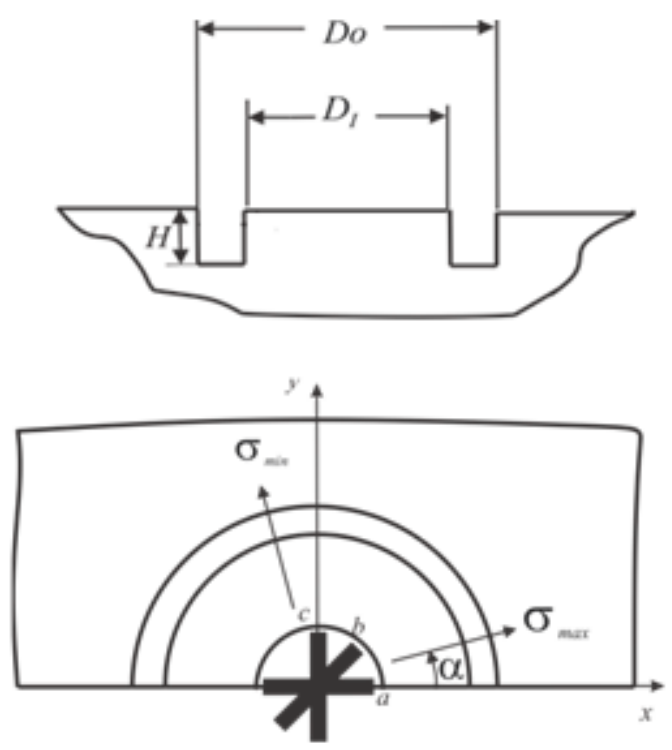

Fig. 2. Geometrical notation of the groove

The use of commercial rosettes with angles $45^{\circ}$ between gauges leads to the simplest analytical expressions. As indicated in Fig. 2, Eq. (4) can be written for each of the gauges a, b and c, by considering their directions:

$$
\begin{aligned}
& \varepsilon_{r}^{a}=\bar{A}\left(\sigma_{x}+\sigma_{y}\right)+\bar{B}\left(\sigma_{x}-\sigma_{y}\right) \cos 2 \alpha \\
& \varepsilon_{r}^{b}=\bar{A}\left(\sigma_{x}+\sigma_{y}\right)+\bar{B}\left(\sigma_{x}-\sigma_{y}\right) \sin 2 \alpha \\
& \varepsilon_{r}^{c}=\bar{A}\left(\sigma_{x}+\sigma_{y}\right)-\bar{B}\left(\sigma_{x}-\sigma_{y}\right) \cos 2 \alpha
\end{aligned}
$$

By solving Eq. (7), the principal stresses and their direction will be as follows: 


$$
\bar{A}=\bar{A}\left(E, v, \frac{H}{D_{I}}\right), \quad \bar{B}=\bar{B}\left(E, v, \frac{H}{D_{I}}\right)
$$

Where $\alpha$ is the angle between gauge 1 and maximum stress. Also, $\varepsilon_{r}^{c}, \varepsilon_{r}^{b}$ and $\varepsilon_{r}^{c}$ are calculated strains by the ring form strain gauges in direction a, b and c. $\bar{A}$ and $\bar{B}$ are constant coefficients and could be obtained experimentally or numerically.

\subsection{Constant coefficient $\bar{A}$ and $\bar{B}$}

Whether the RS analysis application involves through-ring or blind-ring, the coefficients $\bar{A}$ and $\bar{B}$ must be determined for calculating the stresses from the relieved strains. In the case of the through ring, reasonable exact values of the coefficients can be obtained for any particular case by analytical methods, if desired. This can be done by integrating the component of strain parallel to the primary strain-sensing axis of the gauge, over the area of the gauge grid. By giving the details of the grid geometry (line width and spacing, number of lines, etc.); slightly greater accuracy may be obtained by integrating along the individual grid lines. This method cannot be applied to blind ring analysis, because closed-form expressions that relate the relieved strains to the RS in terms of ring depth are not available. Compared to the through-ring coefficients, blindring coefficients involve one additional dimensionless variable; namely, the dimensionless ring depth, $H / D_{I}$ (see Fig. 2). Thus, in a generalized functional form, the coefficients can be expressed as Eq. (5). Basically, the calibration procedure involves calculating the rosette strains under the same applied load or calibration stress, $\sigma_{t}$ both before and after cutting the ring. This procedure is essential to ignore the strain relief effect which may happen due to the initial RS relaxation in the specimen calibration. With this technique, the observed strain difference (before and after ring cutting) is caused only by the applied calibration stress, and is individually related to that the mentioned stress. To calculate the coefficients, only the strains in gauges a and c are necessary, since these gauges are known to be aligned with the principal axes of the specimen. Then a load, P, should be applied to the specimen to develop the desired calibration stress $\sigma_{t}$.

With ideal linear elastic behavior and uniaxial loading condition, the values of coefficients $\bar{A}$ and $\bar{B}$ in Eq. (8) were obtained according to following relation. In this relation, $\sigma_{c}$ is applied stress in direction a, and $\varepsilon_{\text {before }}$ and $\varepsilon_{\text {after }}$ are strain before and after ring machining in two perpendicular directions. The obtained coefficients are firmly applicable only for the same material, ring and rosette geometry used in a calibration test.

$$
\begin{aligned}
& \bar{A}=\frac{\left\{\left(\varepsilon_{r}^{a}\right)_{\text {after }}-\left(\varepsilon_{r}^{a}\right)_{\text {before }}\right\}+\left\{\left(\varepsilon_{r}^{c}\right)_{\text {after }}-\left(\varepsilon_{r}^{c}\right)_{\text {before }}\right\}}{2 \sigma_{t}} \\
& \bar{B}=\frac{\left\{\left(\varepsilon_{r}^{a}\right)_{\text {after }}-\left(\varepsilon_{r}^{a}\right)_{\text {before }}\right\}-\left\{\left(\varepsilon_{r}^{c}\right)_{\text {after }}-\left(\varepsilon_{r}^{c}\right)_{\text {before }}\right\}}{2 \sigma_{t}}
\end{aligned}
$$

\subsection{Plasticity effects on Residual Stresses measurement}

When the specimen material has elastic-plastic behavior, the measured strain is not only affected by the RS relaxation effect, but includes the contribution of other effects such as plastic strain. In particular, it can be given by the following relation:

$$
\varepsilon_{m}=\varepsilon+\Delta \varepsilon_{p}+\Delta \varepsilon_{c}
$$


In which $\varepsilon, \Delta \varepsilon_{p}$ and $\Delta \varepsilon_{c}$ is the strain component due to the residual stresses, the strain component due to a localized plastic flow and the strain component caused by machining the groove, respectively. Machining strain can be minimized by convenient tool and machining method selection, but plastic strain due to material behavior may cause an error in stresses evaluation.

The plasticity error due to stress concentration effect is a well-known issue in the measurement of high residual stresses with the mechanical stress relaxation technique (Gibmeier, Kornmeier et al. 2000). So, for the cases in which the amount of the calculated stress becomes greater than the validity amount, the plastic deformation effects should be taken into account (Moharami and Sattari-Far 2008).

The problem for hole-drilling method has been investigated by many researchers for different cases, during the last two decades (Schajer 1988, Schajer 1988). Procter and Beaney reported $10 \%$ overestimated plastic errors when bars are subjected to full yielding uniaxial loading (Procter and Beaney 1982). Beghini studied a plate with pre-existing hole under mechanical loading to propose a procedure for RS measurement above $50 \%$ of the yield strength (Beghini, Bertini et al. 1994, Beghini and Bertini 1998).

\section{Finite element analysis}

To study the plasticity effects, because of difficulties in experimental research, a finite element method (FEM) was chosen to study the problem. It is the only reasonable way to obtain the desired information or simulate the real experiment. For this purpose, the RC process was simulated by using a commercial finite element code. The ANSYS analysis system was used for the FE-simulation and a parametric model was developed. To ensure the independency of the solution results, the global sizes of the model were selected relatively larger than the ring dimensions. Because of the symmetry in geometry and loading, in order to reduce the required solution time, only one quarter of a finite plate was modeled. The FEM model consisted of about 7500 solid elements. The model and its meshing used for numerical study are depicted in Fig. 3 .
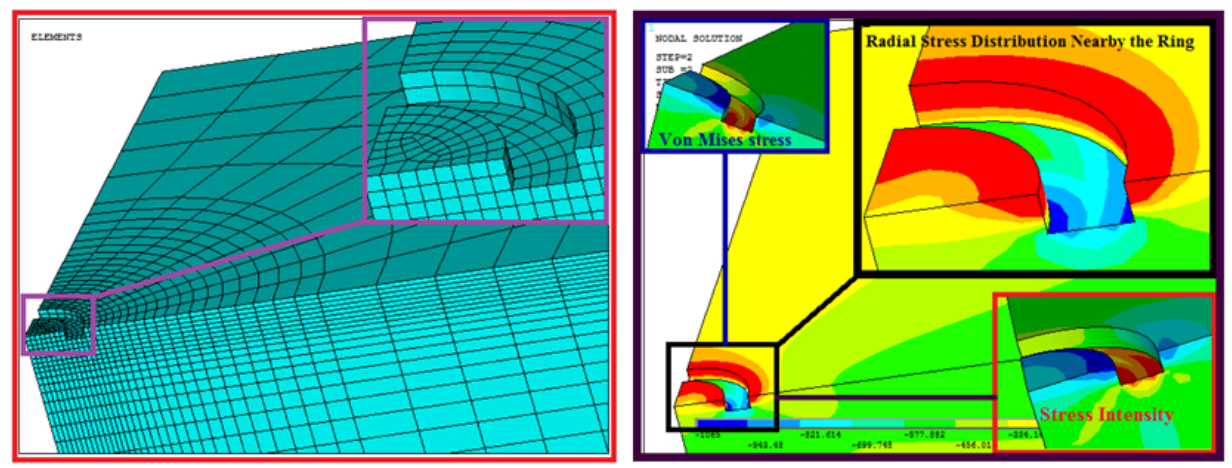

Fig. 3. FEM model used in numerical study of RC method and some schematic results

In this study, by using the killed element technique, machining of a preloaded plate is simulated (2014). By using this model, the relaxed strains for a uniform stress field are obtained. The FEM strain gauge responses were evaluated by averaging strains in the nodes locating under the strain gauges. The accuracy of the numerical solution was checked by using a model with a finer mesh for FE calculation. The difference between the results obtained from the present model and the finer model are negligible for any point of the model. 
To study the plasticity error in the numerical simulation, the assigned mechanical properties are similar to properties of the steels used in the experiments (previously determined by a tensile test). The true stress-strain curves of the materials are modified by bilinear stress-strain behavior which can be easily recognized by the young modulus $E$, yield strength $\sigma_{Y}$, tangent modulus $E_{p}$ and Isotropic strain hardening condition as shown in Fig. 4.
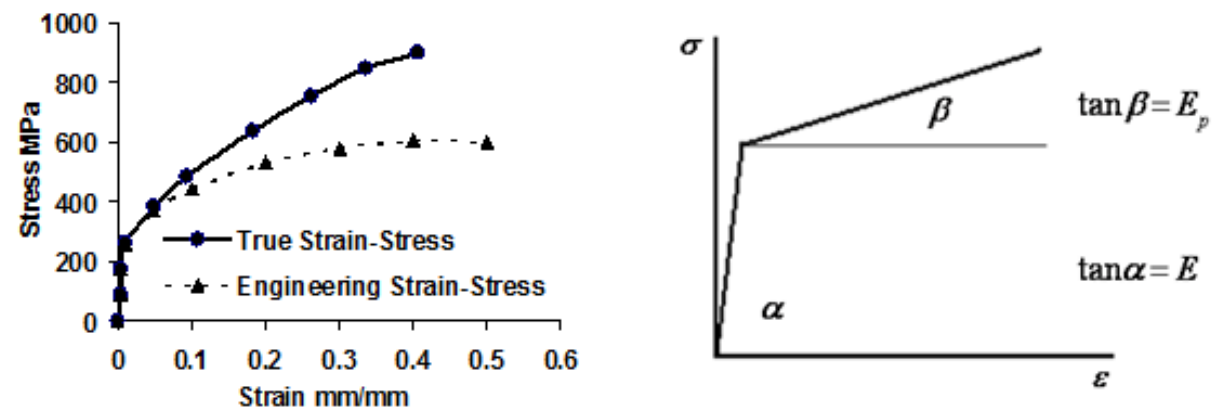

Fig. 4. Approximation of true stress-strain curve of St. St. with bilinear stress-strain curve for FEM analysis.

The plastic error due to yielding around the ring is calculated by subtracting the applied stress $\sigma_{a p p}$ from the calculated stress $\sigma_{c a l}$, divided by the applied stress as expressed by following relation:

$$
\text { Plastic Error }(\%)=\left(\frac{\sigma_{c a l}-\sigma_{a p p}}{\sigma_{a p p}}\right) 100
$$

There are some affecting parameters in the plasticity errors determined by the measured results. Ring geometry, material properties, principal stress level and the stress ratio are the main factors. According to numerical studies, plasticity errors are influenced by the following dimensionless parameters: $P_{1}=H / D_{I}, P_{2}=\sigma / \sigma_{Y}$ and $P_{3}=E_{P} / E$. For studying the effects of these parameters, some finite element analyses were conducted. Through this analysis, in consideration of available rosette strain gauge and machining facility, the internal ring dimension was equal to $10 \mathrm{~mm}$. In these analyses, the effects of hardening coefficient $\left(P_{3}\right)$ and the residual stress level $\left(P_{2}\right)$ for the uniaxial stress field were studied. The values of the affecting parameters studied here are given in Table 1.

\begin{tabular}{|c|c|}
\hline Parameter & Assigned Values \\
\hline$P_{1}=H / D$ & $0.1,0.2,0.3,0.4,0.5$ \\
\hline$P_{2}=\sigma / \sigma_{Y}$ & $0.5,0.55,0.6,0.65,0.7,0.75,0.8,0.85,0.9,0.95$ \\
\hline$P_{3}=E_{p} / E$ & $0.01,0.05,0.10$ \\
\hline
\end{tabular}


Table 1. The values of the affecting parameters used in this study.

\section{Results and discussion}

Using the values form Table 1, the influence of dimensionless parameters $P_{1}, P_{2}$ and $P_{3}$ on the plasticity error were investigated. By Calculating the FEM strain and introducing the applied stresses and calculated stresses to Eq. (11), the plastic errors can be determined. The obtained results for the material with $E_{p} / E=0.05$ are shown in Fig. 5.

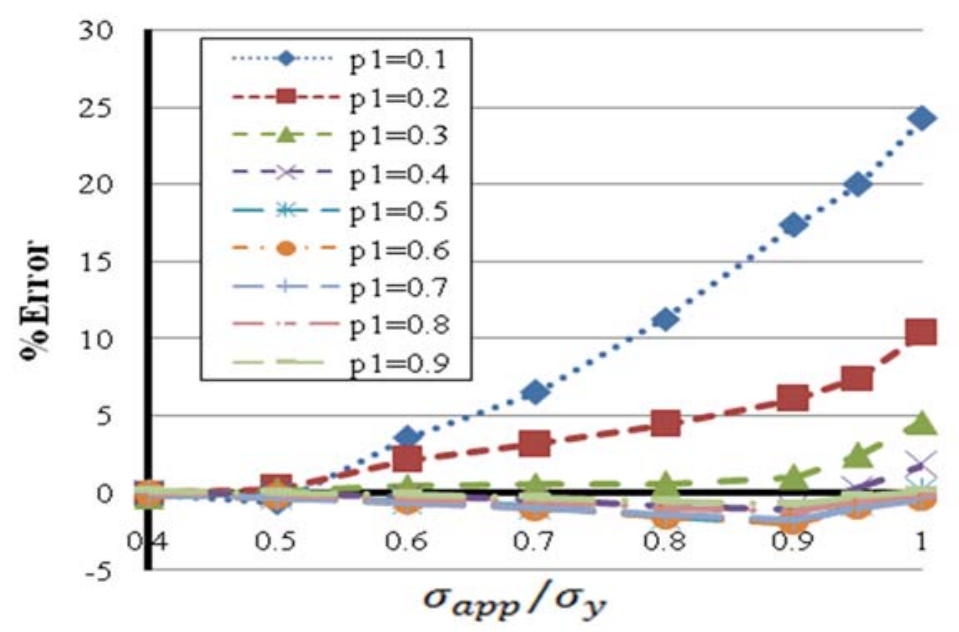

Fig. 5. Effect of the parameter $P_{1}$ on plastic error for $E_{p} / E=0.05$

For materials with the value of $E_{p} / E$ as given in Table 1, the errors in measured stresses have similar behavior as shown in Fig. 5, but with different error orders. According to the results, the plasticity error is negligible for calculated stresses being less than $60 \%$ of the yield strength of the material. When the calculated stress becomes greater than $60 \%$ of the yield strength, errors in the measured results will be significant. The amount of the error increases by increasing the calculated stress.

Based on the figures, the effect of plasticity on RS errors calculating is significant only for surface stresses. Then, recommended depth should be at least $H=0.3 D_{I}$ which corresponds to $P_{1}=0.3$. Also, as $P_{3}$ decreases, the maximum error increases due to tending behavior of the material to a linear elastic state. Therefore, the plasticity error in a uniaxial stress field can be determined as below:

$$
\% \text { Error }=f\left(P_{1}, P_{2}, P_{3}\right)
$$

As can be see from Fig. 5, the influence of $P_{1}$ on the plastic error is significant only for surface stresses, then recommended depth should be at least $H=0.3 D_{I}$ which corresponds to $P_{1}=0.3$. Also, as $E_{p}$ decreases, the maximum error increases. By paying more attention to Fig. 5 , it can be found that as the behavior of material tends towards elastic behavior, $E_{p}$ increases 
and the errors decrease. Another point is that, as drilled groove reaches the bottom layer, the plate surface is less affected by the plastic flow and thus the results will be more accurate.

As the final and the most important result, a modified range is proposed for parameter $P_{1}$ for various values of $P_{2}$, in which the amount of error is less than $1.5 \%$ and can be an acceptable precision for this method (Fig. 6). Then, for the range obtained from this investigation, the method is modified rather than the results presented by Petrucci and Zuccarelio. They reached 40 \% maximum error for the RC method (Petrucci and Zuccarello 1996), while the result of this paper for the maximum error is about $26 \%$.

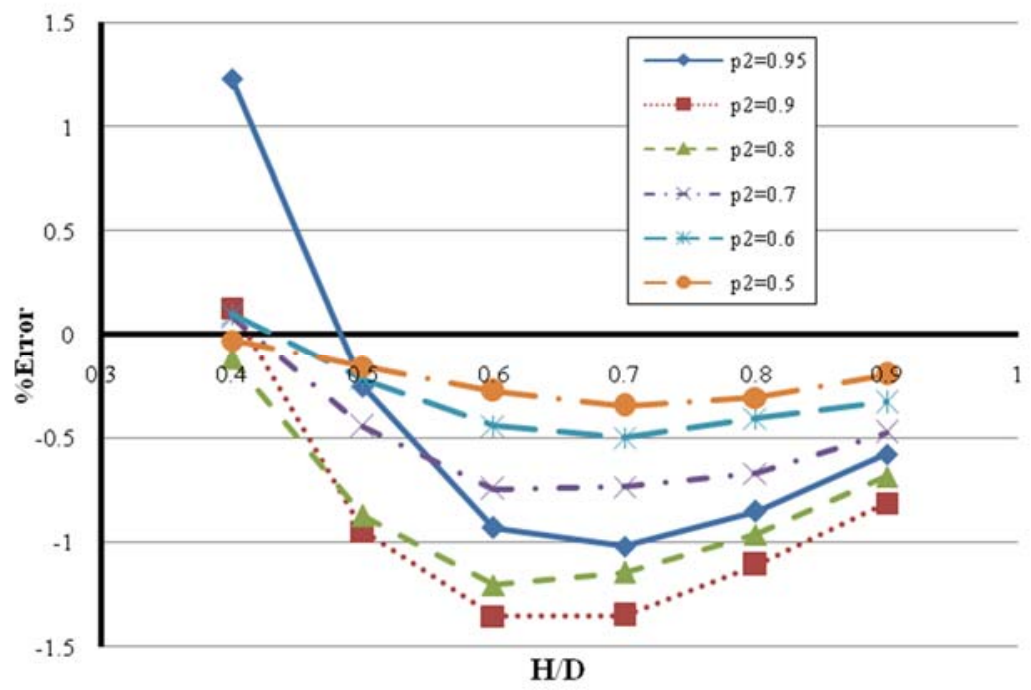

Fig. 6. Modified rings depth Errors

\section{Conclusion}

In this paper, the RC method was initially developed for the uniform RS calculation and then the plasticity effects on the errors' results of this method are investigated. Four parameters are considered in order to study their effect on the precision of results. The results show that, for the case of plasticity effects, there are the following considerations:

- When the uniaxial RS exceeds more than $65 \%$ of the yield strength, for which $P_{2}=0.6$, the error increases.

- Three dimensionless parameters $P_{1}, P_{2}$ and $P_{3}$, also effect the accuracy of the $\mathrm{RC}$ technique in uniaxial loading state.

- The proposed range arise from this research, eliminates the imperfections of other methods in measuring RS.

- The results show that the error percentage is affected by $H / D$, and the accuracy of the method increases in a modified range of this parameter, $0.4 \leq P_{1} \leq 0.9$. 
- The result for the mentioned range has approximately $1.5 \%$ error which is reasonable for residual stresses using the RC method. This is the most important result of this paper.

- The maximum error for this method is about $26 \%$ which is less than the error presented by Petrucci and Zuccarelio that was $40 \%$ (Petrucci and Zuccarello 1996).

\section{References}

Ansys tutorials (2014).

Beaney E, Procter E (1974). A critical evaluation of the centre hole technique for the measurement of residual stresses. Strain, 10(1), 7-14.

Beghini M, Bertini L (1998). Recent advances in the hole drilling method for residual stress measurement. Journal of Materials Engineering and Performance, 7(2), 163.

Beghini M, Bertini L, Raffaelli P (1994). Numerical Analysis of Plasticity Effects in the HoleDrilling Residual Stress Measurement.

Gibmeier J, Kornmeier M, Scholtes B (2000). Plastic deformation during application of the holedrilling method. Materials science forum, Trans Tech Publ.

J. L (1996). Handbook of Measurement of Residual Stress, Society for Experimental Mechanics.

Mathar J (1934). Determination of initial stresses by measuring the deformation around drilled holes. Trans. ASME, 56(4), 249-254.

Moharami R, Sattari-Far I (2008). Experimental and numerical study of measuring high welding residual stresses by using the blind-hole-drilling technique. The Journal of Strain Analysis for Engineering Design, 43(3), 141-148.

Nobre J, Kornmeier M, Dias A, Scholtes B (2000). Use of the hole-drilling method for measuring residual stresses in highly stressed shot-peened surfaces. Experimental mechanics, 40(3), 289-297.

Petrucci G, Zuccarello B (1996). Effect of plasticity on the residual stress measurement using the groove method. Strain, 32(3), 97-104.

Procter E, Beaney EM (1982). Recent Developments in Center-hole Technique for Residualstress Measurement. Experimental Techniques, 6(6), 10-15.

Rendler N, Vigness I (1966). Hole-drilling strain-gage method of measuring residual stresses. Experimental mechanics, 6(12), 577-586.

Rossini N, Dassisti M, Benyounis K, Olabi A (2012). Methods of measuring residual stresses in components. Materials \& Design, 35, 572-588.

Schajer G (1988). Measurement of non-uniform residual stresses using the hole-drilling method. Part I-Stress calculation procedures. Journal of Engineering Materials and Technology, 110(4), 338-343.

Schajer G (1988). Measurement of non-uniform residual stresses using the hole-drilling method. part II-practical application of the integral method. Journal of Engineering Materials and Technology, 110(4), 344-349.

Withers P, Bhadeshia H (2001). Residual stress. Part 1-measurement techniques. Materials science and technology, 17(4), 355-365. 